\title{
Protection at Portal of Entry (PPE) with Povidone lodine for COVID-19
}

\author{
Sumita Shankar ${ }^{1}$, Arunava Saha ${ }^{2, *}$, Limalemla Jamir ${ }^{3}$, Rakesh Kakkar ${ }^{3}$
}

Sumita Shankar ${ }^{1}$, Arunava Saha ${ }^{2, *}$, Limalemla Jamir ${ }^{3}$, Rakesh Kakkar ${ }^{3}$

'Plastic Surgery, Rangaraya Medical College, Guntur, Andhra Pradesh, INDIA. ${ }^{2}$ Christian Medical College, Vellore, Tamil Nadu, INDIA.

${ }^{3}$ Department of Community and Family Medicine, AlIMS Mangalagiri, Guntur, Andhra Pradesh, INDIA.

\section{Correspondence}

Dr. Arunava Saha

Junior Clinical Assistant, Christian Medical College, Vellore-632004, Tamil Nadu, INDIA.

Mobile no: +91-8754923454

Email: saha.arunava100@gmail.com

\section{History}

- Submission Date: 15-05-2020

- Revised Date: 19-06-2020

- Accepted Date: 25-08-2020

DOI : 10.5530/ijmedph.2020.4.36

Article Available online

http://www.ijmedph.org/v10/i4

\section{Copyright}

(C) 2020 Phcog.Net. This is an openaccess article distributed under the terms of the Creative Commons Attribution 4.0 International license.

\begin{abstract}
Background: The current Coronavirus pandemic has infected more than 15 million people worldwide and has been responsible for more than 630,000 deaths worldwide, a significant number of which has been healthcare professionals. Povidone-iodine (PVP-I) has been known as microbicidal agent since the past few decades and it has also demonstrated better anti-viral activity than other antiseptics in a few in vitro studies against other coronaviruses (SARSCoV and MERSCoV). Povidone iodine has also been found to be a safe and effective solution to be used as a gargle or intranasally. Aim and Objectives: Through our study, we would like to propose the application of PVP-I in appropriate and safe concentration through nasal application and oropharyngeal wash to limit the spread of the virus from infected patients to healthcare workers and thus reduce the transmission of COVID-19. Methods: This application is to be done according to the proposed protocol, which has been devised keeping in mind the feasibility, safety and efficacy of PVP-I application. This should be used regularly by patients with a suspected or confirmed COVID-19 infection to not only prevent the spread, but also by healthcare workers prior to treating such patients, or performing perioral or nasal procedures during the pandemic, regardless of the status of the patient. Patients undergoing such procedures should also be treated with PVP-I. The total iodine exposure according to the proposed protocol has been found to be within previously recorded safe limits. Conclusion: We hypothesize that usage of PVP-I as a personal protective equipment at the portal of entry would help decrease the rates of transmission of the SARS-CoV-2 infection.
\end{abstract}

Key words: Betadine, Coronavirus, Gargle, Povidone lodine, Pandemic.

\section{BACKGROUND}

The COVID-19 pandemic, caused by the novel coronavirus SARS-CoV-2, an enveloped nonsegmented positive-sense RNA viruses, belonging to the family Coronaviridae and the order Nidovirales, has infected more than 15 million people worldwide and has been responsible for more than 630,000 deaths. ${ }^{1}$ Significant portion of those affected have been healthcare workers, with a Chinese study showing that this particular group represents about 4 percent of the infected cases, so far about 1700 bedside clinicians being infected. ${ }^{2,3}$ These are dynamic numbers and are constantly increasing on an hourly basis.

A severe supply-demand mismatch for proper personal protective equipment, triggered by panic buying, misuse and hoarding, has led to the frontline workers being dangerously ill-equipped while taking care of such infected patients. ${ }^{3}$ This has placed an unforeseen extra burden on the healthcare system at such a crucial time due to lack of health personnel who are themselves succumbing to the infection. Additionally, a significant risk has been found for non-infected patients who are already hospitalised and remain in close proximity to these infected patients, due to a suspected nosocomial transmission. An Intensive care unit carries one of the highest risks of transmission, as procedures such as non-invasive ventilation, intubation and suction have been known to generate tremendous amounts of bio-aerosol. These particles remain suspended in air and viable on surfaces for longer duration and have much higher chances of causing contact transmission to healthcare professionals and other patients. ${ }^{3}$ Patient saliva has also been found to have a high viral load in COVID-19, ${ }^{4}$ and it is the need of the hour to ensure adequate prevention and prophylaxis for healthcare professionals to prevent further spread of the disease amongst them. In a more recent study it was noticed that the nasopharynx appears to have a higher viral load than that found in the oropharynx. ${ }^{4,5}$ The recommended diagnostic testing requires collection of nasopharyngeal and oropharyngeal swab specimens that necessitates close contact between healthcare workers and the patients. ${ }^{4}$

According to the CDC, in-depth knowledge of the portals of entry and modes of transmission can help to develop appropriate control measures, which are usually directed against the segment in the infection chain that is most susceptible to intervention, which

Cite this article : Shankar S, Saha A, Jamir L, Kakkar R. Protection at Portal of Entry (PPE) with Povidone lodine for COVID-19. Int J Med Public Health. 2020;10(4):166-8. 
is the initial contact in case of this pandemic. ${ }^{6}$ Hence, social distancing and use of masks and other personal protective equipment has been made mandatory, but that has not stopped the spread of the virus. A recent study found that the coronavirus requires ACE2 and TMPRSS2 receptors to enter human body cells. ACE2 is a receptor protein to which SARS-CoV-2 anchors itself. Thereafter, TMPRSS2 activates entry of the virus into a body cell. The goblet cells and the ciliated epithelium in the nose makes it extremely susceptible to the novel coronavirus as they have a very high density of the facilitating protein. ${ }^{7}$ They are also found in good numbers in the corneal cells of human eyes, oesophagus, ileum and colon, which is responsible for the presentation of the infection with diarrhoea. The mouth, being a common passage linking the nose and the lungs, also makes it an important point of entry. And the prevalent human behaviour of frequently touching different parts of the face also promotes dissemination of the virus. ${ }^{8}$

In view of a probable upcoming chance of community transmission, shielding the primary portals of entry, namely the nasopharynx and oropharynx with a mask can be effective, but it is cumbersome to use it all the time. Hence, using an effective virucidal agent, is a simple but effective measure which can be enforced to decrease further spread of this virus.

\section{Povidone lodine and its efficacy}

Povidone Iodine (PVP-I) has been well known as a broad spectrum microbicide against a variety of bacteria, fungi, protozoans and viruses. ${ }^{9,10}$ It was first discovered in 1955 and the active ingredient is listed on the World Health Organization's (WHO) List of Essential Medicines. In a water soluble complex, the elemental iodine is bound to the carrier polyvinylpyrrolidone. A chemical equilibrium develops in an aqueous medium to release the antimicrobial agent iodine, which reacts with the functional groups of amino acids, nucleotides and fatty acids. This leads to disruption of various structures and essential enzymes of microbes and viruses, principal of which was considered to be degeneration of the inner component (nucleoprotein) of the virus particles. ${ }^{11}$

\section{Studies and Clinical Use}

Due to the unique mechanism of action, the development of resistance mechanisms against such a broad oxidative attack looks to be almost impossible. ${ }^{12}$ Also, recent in-vitro studies have demonstrated its efficacy against Ebola virus and two of the coronaviruses that have been responsible for epidemics in the last two decades, namely SARS-CoV causing the severe acute respiratory syndrome (SARS) epidemic of 2002-3 and MERS-CoV the agent responsible for causing the Middle East respiratory syndrome (MERS) epidemic of 2012-13.13-15

The benefit of gargling with PVP-I has also been noted in Japanese clinical respiratory guidelines since $2004 .{ }^{16}$ The initial work was done by Eggers' et al. which looked at the virucidal activity of PVP-I against MERS-CoV. This study demonstrated that the lowest concentration of PVP-I to be effective was $1 \% \mathrm{w} / \mathrm{w}$ when used for $30 \mathrm{sec}$ and led to a reduction of viral activity of $\geq 99.99 \% \cdot{ }^{13}$ In subsequent work by Eggers, lower concentrations were tested and $0.23 \%$ was the lowest concentration which was found to be effective. ${ }^{17}$ Studies done by Kariwa et al have demonstrated that in vitro treatment of SARS-CoV with various preparations of PVP-I for $2 \mathrm{~min}$ was enough to reduce viral activity to undetectable levels. ${ }^{15}$ Similar predictions have been made for surface disinfection to prevent contact transmission with a variety of microbicide agents. ${ }^{18}$ Trials have also successfully proven that nasal inhalation and gargling of PVP-I has reduced the incidence of nosocomial pneumonia by reducing the colonisation of pharyngeal micororganisms. ${ }^{19}$

\section{Safety and tolerance}

Studies have also looked at the prolonged use of PVP-I mouthwash and it was found to be safe, with no significant effect on the thyroid function. ${ }^{20}$ In a study looking at the excretion of iodine, average ingestion of $88 \mathrm{mg}$ per day for a period of 38 days was found to have no adverse effects and primarily cleared through the kidneys. ${ }^{21}$ Staining of the teeth and other mucosal surfaces is minimal, reversible and likely to occur only on prolonged use.

There is a commercially available iodine based 'mouthwash' in India, a $2 \% \mathrm{w} / \mathrm{v}$ mint flavoured solution of PVP-I licensed for oral mucosal use (Povidone-iodine $2 \% \mathrm{w} / \mathrm{v}$ solution, Betadine, with available effective iodine concentration of $0.2 \% \mathrm{w} / \mathrm{v})$. This is diluted to $1: 1$ using sterile water to yield a $1 \%$ solution or, a $10 \%$ solution of PVP-I is diluted to 1:10 using sterile water to yield a $1 \%$ solution, which will provide a solution with a concentration equivalent to $1 \mathrm{mg} / \mathrm{ml}$ of iodine.

\section{Proposed protocol for application of PVP-I}

\section{Pre-administration}

1. Patients or patient's relatives are informed of the benefits and risks of the proposed treatment verbally. Exclusion criteria will be checked and verbal consent will be taken and documented. 2. Healthcare professionals will be offered the administration as a form of PPE, to be used mandatorily while being in contact with suspected or confirmed SARS-CoV-2 infected patients, along with other infection prevention control measures such as hand hygiene, use of protective eye wear, face masks to avoid direct contact with the patient's bodily fluids, as per Indian Council of Medical Research (ICMR) National guidelines in India.

\section{Exclusion criteria}

A. A history of allergy to PVP-I or its any related microbicidal solutions.

B. All forms of thyroid disease.

C. Current ongoing or history of radioactive iodine treatment.

D. Current ongoing or history of Lithium therapy

E. Known pregnancy.

Method of application for conscious patients and healthcare professionals

- Step 1 - For all patients/ healthcare professionals in the described groups: The 1\% PVP-I solution should be administered into each nostril, using an ear bud dipped in the solution, coating as far as possible of the posterior nasopharyngeal wall, or a $0.2 \mathrm{ml}$ solution taken in a syringe and flushed into the nasal cavity, one nostril at a time. The contralateral nostril is occluded by the administrator during the procedure and the patient, if conscious, is asked to inhale slowly during the instillation. This will deliver a total dose of $0.4 \mathrm{mg}$ of iodine.

- Step 2-Conscious patients and healthcare professionals: $10 \mathrm{ml}$ of the $1 \%$ PVP-I solution is introduced into the oral cavity and used as a mouthwash. The solution is distributed throughout the oral cavity for $30 \mathrm{sec}$ and then gently gargled, after which it is held at the back of the throat for another $30 \mathrm{sec}$ before spitting out. It is assumed that at most $2 \mathrm{ml}$ of the solution will be retained and absorbed, giving an anticipated maximum total dose of $2 \mathrm{mg}$ of iodine.

- Step 3 - Unconscious patients. An oral care sponge swab is soaked in $3 \mathrm{ml}$ of $1 \%$ PVP-I and this is carefully wiped around all oral mucosal surfaces during nursing care. Assuming that most of this solution will be retained in the oropharynx due to the above method of application, a maximum total dose of $3 \mathrm{mg}$ of iodine will be absorbed. 


\section{Timing of application of PVP-I}

- Patients hospitalised for confirmed/ suspected COVID-19 and healthcare workers engaged in their care: Steps 1 and 2 should be applicable for patients at every six hourly intervals. The same steps should be undertaken up to four times per day for healthcare workers, with at least a minimum of two hours' interval in between applications. It is also advisable that Steps 1 and 2 are performed prior to contact with the patient/patients and if repeated contact is occurring, repeated every $2-3 \mathrm{hr}$, up to a maximum of 4 times a day.

- Patients undergoing dental procedures, oral surgery, ENT-ORAL examination and treatment, endoscopy and bronchoscopy and any other action to be carried out in the perioral area, oral or nasal cavity

- The patient should undertake steps 1 and 2 prior to the examination or procedure. Healthcare workers conducting the procedure should perform steps 1 and 2 prior to contact with the patient and if multiple patients are being seen, repeat every $2-3 \mathrm{hr}$, up to 4 times a day.

\section{DISCUSSION}

There is considerable evidence of benefit for the use of PVP-I for maintenance of oral hygiene and treatment of oropharyngeal infections. Although studies are not available regarding the effectiveness of PVP-I for controlling the spread of SARS-CoV-2, the available evidence is sufficient to employ its immediate usage as a potential measure in the healthcare setup during this ongoing pandemic. With the current trend of relaxations of the lockdown all around the world and persistently increasing number of cases, regular use of masks and other personal protective equipment may become cumbersome and may not always provide the necessary prevention. In such a scenario, oropharyngeal and nasopharyngeal application of PVP-I, can be done through gargle and intranasal application with cotton buds respectively, to act as a barrier at the primary portal of entry. These procedures are feasible, cheap and does not require any additional active interventions and can be incorporated into the routine personal protective measures adopted by healthcare professionals. Also, there are very few contraindications to using PVP-I and it is also readily available. Hence, we suggest an immediate application of pan-world usage of PVP-I by healthcare workers and their patients to minimise the risk of further spreading of this pandemic.

\section{CONCLUSION}

Oropharyngeal gargle and nasopharyngeal application of PVP-I can be practised by healthcare personnel who come in close contact with COVID-19 patients, or are directly responsible for the management of these patients. Where feasible, COVID-19 patients can also be provided with PVP-I and instructed on using it as a potential barrier and reduction in overall rate of transmission. All patients undergoing perioral procedures, also involving areas around the nose or procedures that transit those areas, including but not limited to a ENT-Oral general examination and treatment, speculum examination, endoscopy, nasopharyngolaryngoscopy, bronchoscopy, elective endotracheal intubation, dental procedures and oral surgery. This should be followed even for the healthcare professional carrying out those procedures. In this regard, the authors have proposed a study to investigate the clinical effectiveness of PVP-I among COVID-19 as briefly mentioned above. To prevent disinformation and stock-out, it is currently being recommended for healthcare settings.

\section{CONFLICT OF INTEREST}

The authors declare no conflict of interest.

\section{REFERENCES}

1. World Health Organization. Coronavirus Situation Report 86. 2020.

2. Surveillances V. The epidemiological characteristics of an outbreak of 2019 novel coronavirus diseases (COVID-19)—China, 2020. China CDC Weekly. 2020;2(8):113-22

3. Wang D, Hu B, Hu C, Zhu F, Liu X, Zhang J, et al. Clinical characteristics of 138 hospitalized patients with 2019 novel coronavirus-infected pneumonia in Wuhan, China. Jama. 2020;323(11):1061-9.

4. To KK, Tsang OT, Yip CC, Chan KH, Wu TC, Chan JM, et al. Consistent detection of 2019 novel coronavirus in saliva. Clin Infect Dis. 2020. ciaa149. doi: 10.1093/ cid/ciaa149

5. Zou L, Ruan F, Huang M, Liang L, Huang H, Hong Z, et al. SARS-CoV-2 viral load in upper respiratory specimens of infected patients. NEJM. 2020;382(12):11779

6. CDC-Principles of Epidemiology in Public Health practice. https://www.cdc.gov/ csels/dsepd/ss1978/lesson1/section10.html

7. Waradon S, et al. Single-Cell Transcriptomics Data Survey Reveals SARS-CoV-2 Entry Factors Highly Expressed in Nasal Epithelial Cells Together with Innate Immune Genes. Nature Medicine. 2020.

8. Kwok YL, et al. Face touching: A frequent habit that has implications for hand hygiene. Am J Infect Control. 2015;43(2):112-4

9. Traore' $\mathrm{O}$, Fayard $\mathrm{SF}$, Laveran $\mathrm{H}$. An in vitro evaluation of the activity of povidoneiodine against nosocomial bacterial strains. J Hosp Infect. 1996;34(3):217-22.

10. Rikimaru T, Kondo M, Kondo S, Oizumi K. Bactericidal activities of povidoneiodine against Mycobacterium. Dermatology. 1997;195(Suppl 2):104-6.

11. Shirai J, Kanno T, Tsuchiya Y, Mitsubayashi S, Seki R. Effects of chlorine, iodine and quaternary ammonium compound disinfectants on several exotic disease viruses. J Vet Med Sci. 2000;62(1):85-92.

12. Schreier H, Erdos G, Reimer K, König B, KönigW, FleischerW. Molecular effects of povidone-iodine on relevant microorganisms: An electron-microscopic and biochemical study. Dermatology. 1997;195(Suppl 2):111-6.

13. Eggers M, Eickmann M, Kowalski K, Zorn J, Reimer K. Povidone-iodine hand wash and hand rub products demonstrated excellent in vitro virucidal efficacy against Ebola virus and modified vaccinia virus Ankara, the new European test virus for enveloped viruses. BMC Infect Dis. 2015:15(1):375.

14. Eggers M, Eickmann M, Zorn J. Rapid and effective virucidal activity of povidoneiodine products against Middle East respiratory syndrome coronavirus (MERSCoV) and modified vaccinia virus ankara (MVA). Infect Dis Ther. 2015;14(4):491501.

15. Kariwa $\mathrm{H}$, et al. Inactivation of SARS Coronavirus by Means of Povidone-lodine Physical Conditions and Chemical Reagents. Dermatology. 2006;212(Suppl 1):119-23

16. Committee for the Japanese Respiratory Society Guidelines in Management of Respiratory. Prevention of hospital-acquired pneumonia (strategies for prevention of hospital-acquired infections). Respirology. 2004;9:S48-50.

17. Eggers $M$, Koburger-Janssen T, Eickmann M, Zorn J. In vitro Bactericidal and Virucidal Efficacy of Povidone-lodine Gargle/Mouthwash against Respiratory and Oral Tract Pathogens. Infectious Diseases and Therapy. 2018;7(2):249-59.

18. Kampf G, Todt D, Pfaender S, Steinmann E. Persistence of coronaviruses on inanimate surfaces and its inactivation with biocidal agents. J Hosp Infect. 2020;104(3):246-51

19. Kawana A Kudo K. A trial of povidone-iodine (PVP-I) nasal inhalation and gargling to remove potentially pathogenic bacteria colonized in the pharynx. Kansenshogaku zasshi. The Journal of the Japanese Association for Infectious Diseases. 1999;73(5):429-36.

20. Ader AW, Paul TL, Reinhardt W, Safran M, Pino SA, Mcarthur WI, et al. Effect of mouth rinsing with two polyvinylpyrrolidone-iodine mixtures on iodine absorption and thyroid function. J Clin Endocrinol Metab. 1988;66(3):632-5.

21. Nelson N, Palmes ED, Park CR, Weymouth PP, Bean WB. The absorption, excretion and physiological effect of iodine in normal human subjects. J Clin Invest. 1947;26(2):301-10. 